\title{
Rapid Response and Robotic Telescopes For Understanding Small Body Transient Science
}

Tim Lister ${ }^{1}$, Las Cumbres Observatory (tlister@lco.global, 805880 1989)

Michael S. P. Kelley², Geza Gyuk ${ }^{3}$, Quanzhi Ye², Jian-Yang Li ${ }^{4}$

${ }^{1}$ Las Cumbres Observatory (LCO), Goleta, CA, USA

2University of Maryland, College Park, MD, USA

${ }^{3}$ Adler Planetarium, Chicago, IL, USA

${ }^{4}$ Planetary Science Institute, Tucson, AZ, USA
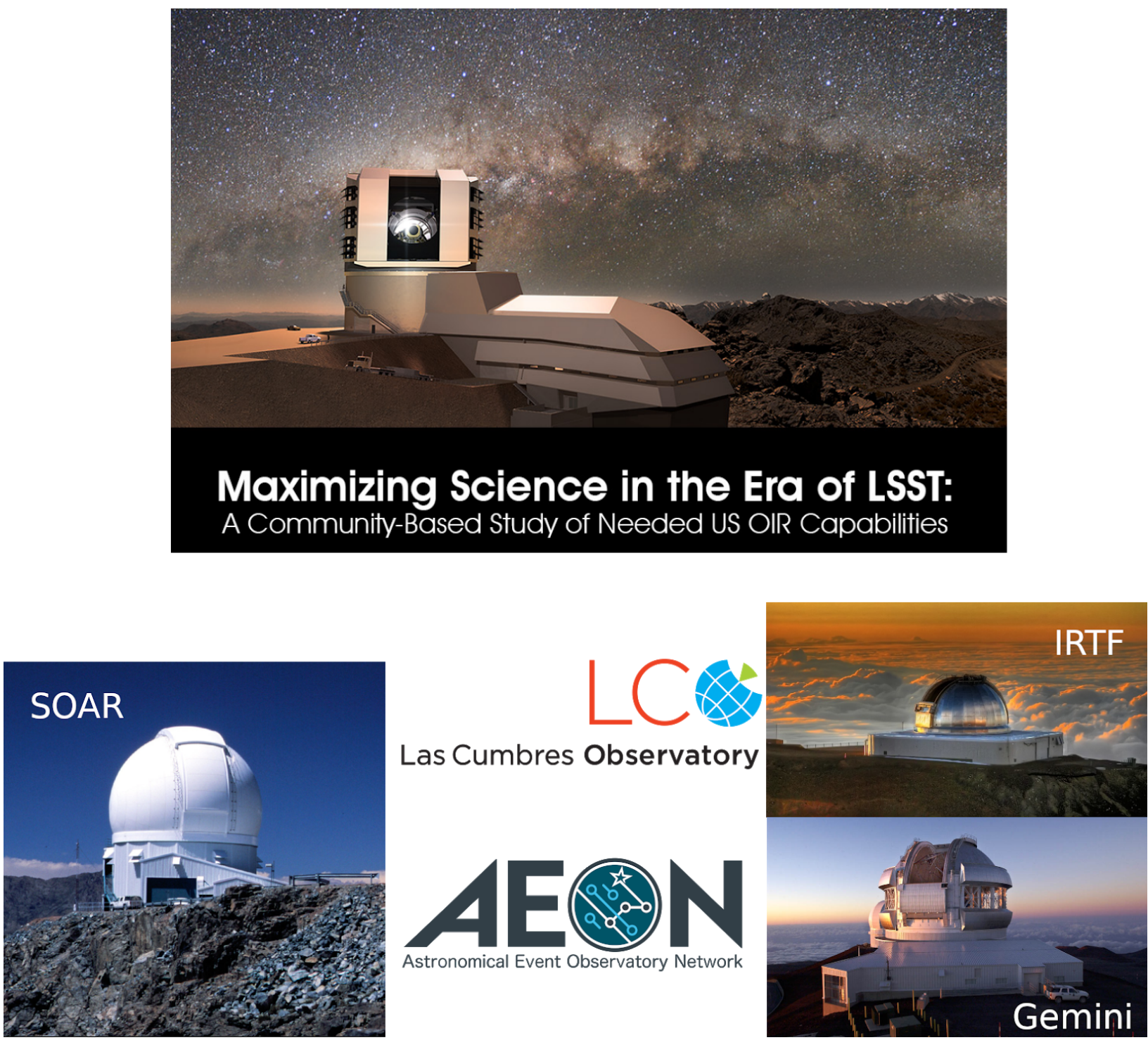


\title{
Rapid Response and Robotic Telescopes For Small Body Transient Science
}

\begin{abstract}
Existing sky surveys are detecting a wide range of transient phenomena in the solar system and are opening up a fascinating time-domain window on planetary science. This will increase considerably with future sky surveys such as the Vera C. Rubin Observatory's Legacy Survey of Space and Time (LSST) which will carry out the survey throughout the period of this decadal survey. Exploiting these exciting science opportunities requires a range of flexible facilities that are capable of rapid response and a matching commitment for rapid response and high throughput at all stages of the follow-up chain. This extends from the surveys through alert brokers, follow-up facilities, data processing and archiving to the end-user scientists. This must be matched with acknowledgement and support for the vital role played by developers of the needed instrumentation and software in enabling all planetary science to take place, but in particular these systems that will be needed for high volume of transients from future surveys. In this white paper, we outline some of the time-domain planetary science that can be unlocked with robotic telescopes and rapid response follow-up facilities.
\end{abstract}

\section{Introduction}

Asteroids and comets are small bodies left over from the formation of the Solar System. Their preserved interiors and exteriors hold key information on the conditions and processes extant at that early epoch and the processes that have shaped the Solar System since then. Understanding the properties of small bodies in our Solar System is key to understanding our own origins as well as giving context to our searches for volatiles and the precursors to life in the large number of exoplanetary systems now being discovered. The subset of asteroids that can approach and potentially threaten the Earth, the Near Earth Objects (NEOs), also create demands for a rapid response to characterize them to the fullest extent possible during potentially brief windows of observability. NEOs are our closest neighbors and research into them is important in order to understand the origin and evolution of the Solar System, but also to understand and protect human society from potential impacts.

Although comets have spent most of the time unchanged in the outer solar system, despite this primitive nature, comets do evolve. Once in the inner Solar System, heating by the Sun will preferentially cause the loss of the most volatile components (e.g., Prialnik \& Rosenberg, 2009), while activity itself also drives both physical changes (Jewitt 2004) and chemical changes (e.g., Feaga et al 2007; A'Hearn et al. 2011; Le Roy et al. 2015) in cometary nuclei. Understanding this evolution is crucial to connecting observed cometary properties back to conditions in the early solar system, which can then be used to constrain elements of Solar System dynamical evolution models like the Nice and Grand-Tack models (Levison et al., 2009; Walsh et al., 2011). Studies of comets and cometary outbursts have shown that there can be rapid, dynamic changes on a wide variety of timescales. Study of these rapid phenomena can help greatly in working towards understanding the structure and evolution of comets.

There is also a growing understanding that at least some asteroids are dynamic objects. In recent years, the discovery of active asteroids, including main-belt comets and 
disrupted asteroids (e.g., the activity of (6478) Gault detected by the ATLAS and ZTF surveys; Ye et al. 2019), have brought greater interest to this area, and studies of these objects can bring additional insights into the evolution of the different subpopulations of small bodies in the Solar System (Jewitt et al. 2015). Active asteroids appear to be a diverse population driven by a variety of mechanisms including volatile sublimation, direct impacts, and rotation-driven mass shedding. These mechanisms are central to our understanding of the processes shaping the evolution of asteroids as a whole. Characterizing active asteroids is a direct probe of a crucial part of Solar System history.

\section{Science from Small Body Transients and Outbursts}

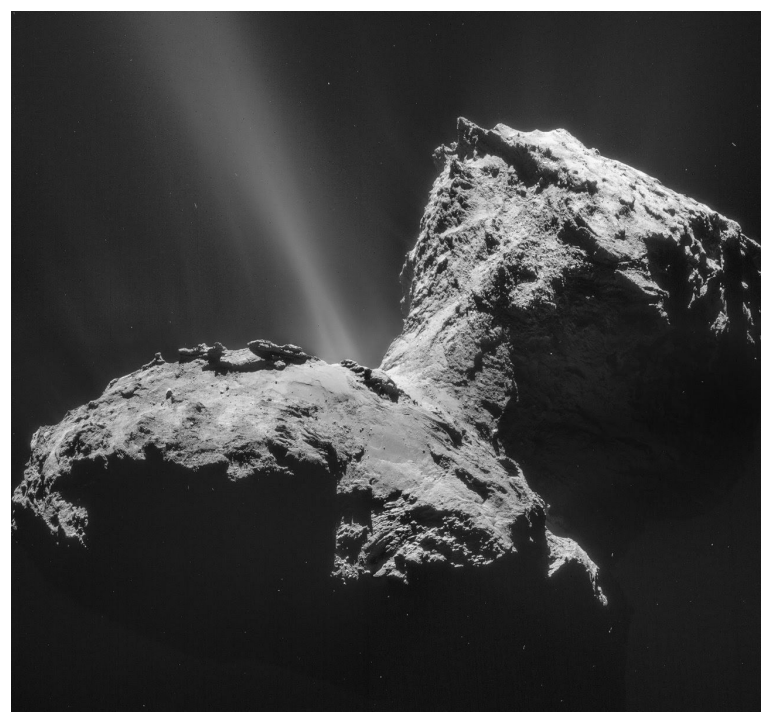

Solar System object outbursts are sudden, short-lived mass-loss events which can span many orders of magnitude in ejection mass and outburst frequency. They vary from small scale outbursts every few rotation periods (seen by the Deep Impact and Rosetta spacecraft at 9P/Tempel 1 and 67P; e.g., Belton et al. 2013, Vincent et al. 2016, Figure left) to large scale fragmentation or complete disruption of the comet (Hughes 1990, Ishiguro et al. 2016). In recent years, the discovery of active asteroids, which include main-belt comets and disrupted asteroids, have brought greater interest to this area and study of these objects can bring additional insights into the evolution of the different subpopulations of small bodies in the Solar System (Jewitt et al. 2015). A variety of mechanisms have been put forward to explain the outburst process (Hughes 1991). These include the uncovering of pockets of ices with low vaporization temperature as the comet heats up on the way to perihelion. On dynamically new comets, outbursts may also be triggered by the exothermic conversion of amorphous water ice to crystalline ice (cf. Prialnik and Bar-Nun 1992). For asteroids with a diameter $\sim 1 \mathrm{~km}$, spinup via the YORP effect can occur on timescales as little as a million years. As spin rates approach the critical value, sudden structural failure and mass shedding can occur. Even for larger asteroids, small changes in the spin state can rearrange the surface, producing dust events or revealing hidden volatiles.

While asteroids have generally been thought to be dry, the discovery of ice in a wide range of environments (such as the surfaces of the Moon and Mercury) has provoked re-examination of this issue. Careful modeling suggests that, depending on the model, distance from the Sun, and regolith parameters, ice may survive for billions of years at depths anywhere from just meters to hundreds of meters. Ice-bearing asteroids may therefore be "primordial", or implanted later as objects which formed further out are transported by resonances and encounters with major planets. While deeply buried ice 


\section{Rapid Response and Robotic Telescopes For Small Body Transient Science}

would not be expected to be sublimating at detectable rates, any process that excavates the ice (impacts, surface/morphology rearrangements etc.) would trigger months-long episodes of sublimation driven activity, especially around perihelion.

The so-called Main Belt Comets appear to be just such a class of objects. The first discovered was 133P/Elst-Pizarro, which in 1996 was seen to be sporting a long, thin dust tail. It is believed that this dust emission is caused by the vaporization of volatiles such as water ice and not an instantaneous event such as an impact. Since 1996, additional main belt comets have been discovered, with roughly half of them belonging to the Themis dynamical family of asteroids. The Themis family, with several thousand members, is believed to have formed in the catastrophic collisional breakup of a several hundred $\mathrm{km}$ wide parent body about 2 billion years ago. Themis family members have (broadly) C-type reflectance spectra, interpreted as similar to carbonaceous chondrite meteorites. Such meteorites have undergone aqueous alteration by liquid water. It is thought that Themis family members, as fragments of a large water-rich parent body, may still reveal the presence of ice. The discovery and characterization of additional main belt comets, by short- and long-term imaging, has the potential to greatly improve our understanding of the presence of water in asteroids.

Another class of active asteroids are those in which their activity was initiated by a collision. The dust release would be relatively instantaneous, and would be distinguished from main belt comets by the modeling of long-term dust emission. Additionally, high-resolution imaging of the pattern of dust emission shortly following an impact may reveal information about the size and impact parameters of the impacting object, since the structure of the dust cloud would be dominated by impact effects rather than longer-term dynamical effects from radiation pressure. The discovery and analysis of impact-produced activity could provide important constraints on the population of very small asteroids in the main belt.

A third class of objects are those whose activity results from mass shedding due to rotational instability. These parent asteroids would exhibit relatively short and perhaps repeated periods of dust emission. The parent bodies are seen to be rotating at near the gravitational breakup rate (with periods of around 2.3 hours), and would be distinguished from the other types of active asteroids both on the basis of their rotation periods (determined by lightcurve observations) and the analysis of their dust emission patterns. For asteroids with a diameter of $1 \mathrm{~km}$, spinup via the YORP effect can occur on timescales as little as a million years. As spin rates approach the critical value sudden structural failure and mass shedding can occur. Even for larger asteroids, smaller changes in the spin state can produce rearrangements of surface regolith, potentially producing dust or revealing hidden volatiles. Direct observations of Ryugu and Bennu by the Hayabusa2 and OSIRIS-REx spacecraft not only confirm the global rearrangement of surface materials, but have actually caught continuous ejection of particles from Bennu's surface, indicating that this phenomenon may be widespread.

Although estimates of active asteroids in the general population cover a wide range in frequency from perhaps $10^{-4}$ to as rare as $10^{-6}$, and to date only around a few dozen 


\section{Rapid Response and Robotic Telescopes For Small Body Transient Science}

active asteroids have been found, current and deep future surveys such as ZTF and LSST will almost certainly increase these numbers by orders of magnitudes.

\section{A Growing Need \& Capability For Rapid Response Small Body Transient Science}

Current sky surveys, such as ATLAS (Tonry et al. 2018), CSS, PanSTARRS (Chambers et al. 2016) and the Zwicky Transient Factory (ZTF; Bellm et al. 2019), and future surveys such as the LSST (Ivezić et al. 2019), allow whole sky monitoring on timescales of a few days. These surveys discover many new NEOs and monitor many tens of known comets and thousands of known asteroids every night, providing transient alerts within minutes - hours. These alerts enable and require rapid response follow-up from other telescopes to characterize the outburst on timescales that are much shorter than the survey cadence ( 2 to 3 days).

The increasing amount of the sky that is being monitored on a nightly basis by the various synoptic surveys means that many previously undetected outbursts of comets and asteroids will be detected and alerts generated shortly after the outburst. This in turns drives the nature of the follow-up and the resources used to be able to respond quickly, confirm and monitor the outburst over an extended period of time and co-ordinate and collate the wide variety of the data that will be produced.

Target and Observations Manager (TOM) systems are designed to coordinate programs where the workload of keeping track of targets, observations and data products would otherwise be onerous. They provide a framework for science-specific analysis to be conducted and to interact with external services to receive alerts and targets, submit follow-up requests to telescope facilities and receive data and feedback status from these facilities. The TOM Toolkit (Street et al. 2018), developed by LCO, allows TOM systems to be built more easily by providing a large set of core functions for commonly-used software components and providing well-defined interfaces to allow science-specific code to interact with the TOM. The open source and publicly available TOM Toolkit ${ }^{1}$ is being developed by LCO with community input.

The Astronomical Events Observatory Network (AEON) ${ }^{2}$ aims to bring together a network of observatories to allow time-domain follow-up and science to be performed in a rapid, efficient and homogeneous way. It adds the SOAR 4.1-m telescope and Goodman spectrograph (optical) and the Gemini South 8-m telescope and likely the SCORPIO instrument (optical-NIR imager/spectrograph) once commissioned to the existing LCO telescope network of $0.4,1$, and 2-m telescopes, creating a more powerful "virtual facility". This "virtual facility" becomes a powerful tool for the study of solar system object outbursts and their subsequent evolution with access to telescope resources from $0.4-8-\mathrm{m}$ with optical and NIR imaging and low resolution spectroscopy instruments. The higher spatial resolution and greater light gathering power of the larger

${ }^{1}$ https://tomtoolkit.github.io

${ }^{2}$ https://lco.global/aeon 


\section{Rapid Response and Robotic Telescopes For Small Body Transient Science}

telescopes allows the study of details of the structure closer in to the nucleus and allows the evolution of the outbursts to be tracked longer as they decay away beyond what can be studied with smaller apertures.

The production of transient alerts from current and future sky surveys, the integration of alert streams from alert brokers such as ANTARES, with the target coordination and follow-up capabilities provided by TOMs, and rapid response telescopes and data processing pipelines allows "closed loop" small body science to be achieved on exciting short timescale dynamic phenomena across the Solar System.

\section{Future Facilities and Resources to Enable Small Body Transient Science}

The 2016 Kavli Report Maximizing Science in the Era of LSST: A Community-Based Study of Needed US OIR Capabilities (Najita et al. 2016) emphasized:

- Broad wavelength range OIR spectrographs on 8-m telescopes in the Southern Hemisphere

- Software tools \& infrastructure (brokers) to handle the alert rate from LSST

- API-accessible brokers, observing and archive facilities

- Access, time allocation \& scheduling policies suitable for time-domain astronomy

- Evolving data sharing practices to allow broader and more effective collaboration on the larger data sets in the coming decade

Schwamb et al. 2019 set out the software requirements for Solar System science needs for LSST in Year 1 (2023, which will also be the first year covered by the new decadal) and later years. Some of these needs include:

- Automatic algorithms to search the LSST alert stream and MOPS detections for cometary activity, catastrophic disruptions, impacts, significant brightening events, and other signs of activity.

- Infrastructure for coordinating and disseminating time critical follow-up observations of NEO discoveries, cometary outbursts, interstellar object flybys, and other timely characterization opportunities.

In addition to these, continued international collaborations is vital to ensure global coverage in both longitude and latitude, ensuring timeliness and quick reaction times for observations. Expansion of the current global networks such as LCO \& AEON, and the addition of more facilities, capabilities and observing sites and stations is needed.

This leads us to our aggregation of future facilities and resources that will be needed:

Continued and increased access to flexibly scheduled small (1-4 m) telescopes: Given the expected single visit exposure depth ${ }^{3}$ for LSST will be $r \sim 24.7$ mag. and transient outbursts can increase this brightness by anywhere from a few tenths to many magnitudes, coupled with engineering practicalities of moving large telescope masses rapidly, results in small telescopes in the 1-4 meter range being the most suitable and practical for the initial follow-up imaging, photometry and spectroscopy (for brighter

${ }^{3}$ https://confluence.Isstcorp.org/display/LKB/LSST+Key+Numbers 


\section{Rapid Response and Robotic Telescopes For Small Body Transient Science}

transients/larger facilities). These facilities will take the lead for the initial characterization of transients since they can respond much more rapidly than larger facilities and before the original survey re-observes the original field. Continued support for these smaller telescopes, along with the addition of enhanced instrumentation such as multi-band imagers (e.g. the SED Machine ${ }^{4}$, MuSCAT2 ${ }^{5}$ ) to increase the throughput of transient response, will be essential to capture the science in the early stages of evolving transient phenomena. They will also be vital to screen and vet transients before triggering larger ( $8+$ meter) facilities where time is more valuable and which will be overwhelmed with astrophysical and planetary transient demands for follow-up.

Rapid response modes on future $\mathbf{1 0}$ - $\mathbf{3 0}$ meter class and space-based telescopes: Although time for planetary transients on the future large telescopes facilities such as the TMT, GMT and ESO's ELT will be extremely limited, the combination of the huge light gathering power and spatial resolution offered will be vital for the in-depth study of some solar system transient phenomena. Space-based facilities, such as HST and the future JWST and other facilities offer access to spectral bands inaccessible from the ground, diffraction limited spatial resolution, high sensitivity in the stable space environment, and observing schedules that are not limited by day-night cycle. HST has proven to be vital in the observations of many such solar system transient phenomena. The short durations (usually days to weeks) of the planetary transients demand flexibility and quick response mechanisms for accessing these facilities. Care should be taken in the design, development and implementation of the hardware, software and observing policies to allow flexible and rapid response to solar system transients.

Additional low resolution optical/NIR spectroscopy on rapid response/robotic facilities: High throughput, broad wavelength coverage optical/NIR spectroscopy is a vital diagnostic tool for almost all planetary (and astrophysical) transient characterization. There is already a critical need for low resolution spectroscopy from the transients already being produced by the existing sky surveys, a need that will only intensify with the start of the Rubin Observatory's survey. Additional low-medium resolution (resolution of a few hundred to a few thousand) spectrographs on 4-8 meter telescopes with queue-observing and rapid response capabilities will be invaluable for the rapid characterization of a wide range of planetary and astrophysical transients.

Equitable access to alerts, archives, and follow-up facilities for all planetary scientists: With the LSST transients alerts being made public to the entire astronomical community within minutes of processing, starting in year 2 of the survey, there is the opportunity to make the exploitation of transient and time domain science more accessible to a broader range of scientists than before. This will allow researchers at smaller institutions without guaranteed or regular access to telescope facilities to perform cutting edge science. While some of this research will be possible with the LSST data alone, full understanding of the new scientific discoveries and insights will only be possible with equitable access to follow-up facilities and additional resources such as high end computing, data archives and multi-wavelength datasets.

Recognition and support for the vital role in performing and enabling planetary science provided by developers and engineers: Virtually all science in recent times

${ }^{4}$ https://www. astro.caltech.edu/sedm/

${ }^{5}$ http://research.iac.es/OOCC/iac-managed-telescopes/telescopio-carlos-sanchez/muscat2/ 


\section{Rapid Response and Robotic Telescopes For Small Body Transient Science}

has been carried out by teams and collaborations with people with expertise and experience in instrumentation, software and hardware development. The instruments, tools, data processing \& analysis software and archiving developed during these projects and collaborations are frequently vital to obtaining and enabling the scientific investigation to take place. Often these projects can span many years or longer. However the traditional academic career and grant funding structures can actively penalize, undervalue and disincentivize people who wish to make contributions to projects in software development and data management roles, which are often seen as less important. There needs to be greater recognition of the vital role software development plays in modern astronomy and planetary science and appropriate advancement and career progression opportunities for people who devote some or all of their time to this area and allow current and future planetary science to happen.

\section{Relevant White Papers}

Ground-Based Radar for Planetary Science and Planetary Defense - Taylor et al.

Main Belt Comets as Clues to the Distribution of Water in the Early Solar System Meech et al.

Planetary Science with Astrophysics Assets - Bauer et al.

The Crucial Role of Ground- and Space-Based Remote Sensing Studies of Cometary Volatiles in the Next Decade (2023-2032) - Roth et al.

The Future of Planetary Defense in the Era of Advanced Surveys - Mainzer et al.

The Scientific Impact of the Vera C. Rubin Observatory's Legacy Survey of Space and Time for Solar System Science - Jones et al.

\section{References}

A'Hearn, M. F., et al., 2011, Science, 332, 1396.

Agarwal, J. et al., 2017, MNRAS, 469, S606.

Bellm, E. C. et al., 2019, PASP, 131, 018002.

Belton, M. J. S. et al., 2013, Icarus, 222, 477.

Chambers, K. C. et al., 2016, arXiv:1612.05560.

Feaga, L., et al., 2007, Icarus, 190, 345.

Hughes, D. W., 1990, QJRAS, 31, 69.

Ishiguro, M. et al., 2016, AJ, 152, 169.

Jewitt, D., 2004, In Comets II, p. 659.

Jewitt, D., Hsieh, H., Agarwal, J., 2015, In Asteroids IV, p. 221.

Levison, H. F., et al., 2009, Nature, 460, 364.

Le Roy, L., et al., 2015, A\&A, 583, 1.

Najita, J., et al., 2016, arXiv:1610.01661.

Prialnik, D., \& Rosenberg, E. D., 2009, MNRAS, 399, L79.

Schwamb, M. et al., 2019, RNAAS, 3, 51, doi: $10.3847 / 2515-5172 / a b 0 e 10$

Street, R. A. et al. Proceedings of SPIE, 10707, 1070711.

Tonry, J., et al., 2018, ApJ, 867, 105.

Vincent, J.-B., et al., 2016, MNRAS, 462, S184.

Walsh, K. J. et al., 2011, Nature, 475, 206.

Ye, Q. et al., 2019, ApJL, 874, 16. 